\title{
Ultraviolet Stability of Three-Dimensional Lattice Pure Gauge Field Theories $\star$
}

\author{
T. Balaban $\star \star$ \\ Department of Mathematics, The University of Michigan, Ann Arbor, MI 48109, USA
}

\begin{abstract}
We prove the ultraviolet stability for three-dimensional lattice gauge field theories. We consider only the Wilson lattice approximation for pure Yang-Mills field theories. The proof is based on results of the previous papers on renormalization group method for lattice gauge theories.
\end{abstract}

\section{Introduction}

In this paper we give the first, simplest application of the results of [2]-[7]. In these we have developed the renormalization group approach to gauge field theories, in the form proposed by K. Wilson in $[24,25]$. We prove here ultraviolet stability of lattice approximations to three-dimensional pure Yang-Mills field theories. We give a simple proof, following the method of [8]-[10] on three-dimensional Higgs model, i.e. a proof using some special features of superrenormalizable models, but we use also Wilson's ideas on the role of scaling transformations. More exactly in lattice gauge theories we use improvement of regularity properties of typical gauge fields instead of non-existing scalings. In fact we use these ideas in the proof of finiteness of the resulting effective theory, and this aspect of the proof is non-perturbative, although we use the superrenormalizability and perturbative expansions to produce this effective theory. This makes the proof especially simple and short. It is based on almost all results of the papers [2]-[7], and we assume that the reader is familiar with these, especially with notations and definitions. Reading [1] is recommended for those who want to get enough information to read the present paper independently of the others.

An awkward and difficult aspect of the paper is a formulation of results. At the beginning we start with a general and simple formulation, and then we make it more precise when we develop our method. Thus let us explain at first in very general terms what we understand by the ultraviolet stability. This notion is strictly connected

* Work partially supported by the National Science Foundation under Grant PHY 82-03669 and DMS 84-01989

** On leave of absence, Postal address. Department of Physics, Harvard University, Cambridge, MA 02138, USA 
with the renormalization group approach. We start with the action density

$$
\rho_{0}(U)=\exp \left[-\frac{1}{g_{0}^{2}} A(U)-E\right]
$$

where $U$ is a gauge field configuration on the torus $T, g_{0}^{2}=g^{2} \varepsilon^{4-d}=g^{2} \varepsilon(d=3), A(U)$ is the Wilson action, and $E$ is a constant including normalization terms and vacuum energy renormalization counterterms. This constant can be defined perturbatively by a finite order expansion of the integral $\int d U$ (gauge fixing term) $\exp \left[-\left(1 / g_{0}^{2}\right)\right.$ $A(U)]$ with respect to $g$, but we prefer to give an inductive definition during the proof. To the density $\rho_{0}$ we apply successively the renormalization transformations $T$ described in $[1,4]$. This yields a sequence of densities $\rho_{k}$ defined inductively by

$$
\rho_{k+1}=T \rho_{k} .
$$

The density $\rho_{k}(U)$ is a function of configurations $U$ defined on the lattice $T^{(k)}$. It is convenient to assume that all the lattices $T^{(k)}$ are unit lattices, but if we rescale the initial lattice $T$ to the $\varepsilon$-scale, i.e. we consider the model on the lattice $T_{\varepsilon}$, then the density $\rho_{k}$ is defined on $L^{k}$-lattice $T_{L^{k}}^{(k)}$. We terminate constructions of the densities $\rho_{k}$ when we reach the unit lattice, or more exactly when $L^{k} \varepsilon=\varepsilon_{0}$, where $\varepsilon_{0}$ is a positive constant depending on the coupling constant $g$ only. Let us denote by $K$ the index satisfying this equality, i.e. $L^{K} \varepsilon=\varepsilon_{0}$. Obviously $K$ depends on $\varepsilon$ and $\varepsilon_{0}$.

The ultraviolet stability means that the actions $\rho_{K}$ have bounds independent of the lattice spacing $\varepsilon$. In our case field configurations have values in the compact Lie group $G$, hence bounds are in uniform norms, and can be written in a simplest way as

$$
\chi(U) e^{-O(1)\left|T_{\varepsilon}\right|} \leqq \rho_{K}(U) \leqq e^{O(1)\left|T_{\varepsilon}\right|}, \quad\left|T_{\varepsilon}\right|=\sum_{x \in T_{\varepsilon}} \varepsilon^{3},
$$

with a constant $O(1)$ depending on $g$ and $\varepsilon_{0}$ only. The function $\chi(U)$ is a characteristic function of the domain

$$
|U(\partial p)-1|<\varepsilon_{1}, \quad p \subset T_{1}^{(K)}
$$

where $\varepsilon_{1}$ is a sufficiently small positive constant, which will be chosen later. The constant $O(1)$ goes to $\infty$ as $g \rightarrow 0$. To get a better bound we have to write explicitly the expression divergent with $g$. It is also convenient to generalize bounds to the whole renormalization group flow, that is to the whole sequence $\rho_{k}, k=0,1, \ldots, K$. Thus the ultraviolet stability for the flow means the sequence of bounds

$$
\chi(U) \exp \left[-\frac{1}{g_{k}^{2}} A^{\eta}\left(U_{k}(U)\right)-O(1)\left|T_{1}^{(k)}\right|\right] \leqq \rho_{k}(U) \leqq \exp O(1)\left|T_{1}^{(k)}\right|
$$

where $U_{k}(U)$ is the minimal configuration constructed in [7] and determined by the configuration $U$ on $T_{1}^{(k)}$, and satisfying (4),

$$
\begin{aligned}
A^{\eta}\left(U_{k}(U)\right) & =\sum_{p \in T_{\eta}} \eta^{-1}\left[1-\operatorname{Re} \operatorname{tr} U_{k}(U, \partial p)\right], \quad \eta=L^{-k} \\
g_{k} & =g\left(L^{k} \varepsilon\right)^{1 / 2}, \quad\left|T_{1}^{(k)}\right|=\sum_{y \in T_{\mathrm{L}}^{(k)}} 1=\sum_{x \in T_{\eta}} \eta^{3}=\left(L^{k} \varepsilon\right)^{-3}\left|T_{\varepsilon}\right|
\end{aligned}
$$


and the constant $O(1)$ is independent of $\varepsilon, k, g_{k}$ in a bounded set.

Now we can formulate the main result of this paper.

Theorem 1. The lattice approximations of the three-dimensional pure Yang-Mills theory with a semi-simple compact group Lie $G$ are ultraviolet stable in the sense that the sequence of densities $\rho_{k}$, constructed by the inductive definition (2), with $\rho_{0}$ given by (1), satisfies the bounds (5).

The above theorem can be generalized to a much wider class of compact Lie groups, as it will be clear from the proof. We have restricted ourselves to semi-simple groups because then the proof is particularly simple.

The bounds (5) imply bounds for partition functions, i.e. for the integrals $\int d U \rho_{k}(U)$, hence by normalization identities

$$
\int d U \rho_{k}=\int d U T^{k} \rho_{0}=\int d U \rho_{0}=Z^{\varepsilon},
$$

they imply uniform in $\varepsilon$ bounds for the partition function $Z^{\varepsilon}$.

In the proof of the above theorem we concentrate mainly on the proof of the upper bound, we make only few remarks about the easier lower bound. The proof is divided into four sections. In the first we describe the procedure in the first renormalization transformation. The second section gives a description of a precise bound for the general density $\rho_{k}$. In the third section we give an inductive proof of this bound. The last, fourth section contains a discussion of the results.

Let us mention finally that the proof is very sketchy and we do not discuss many technical details. It is so because we intend to improve several aspects of the proof, and here we develop only main ideas.

Since the publication of the first papers in this series, i.e. since the Boulder conference, the new papers $[16,17,18]$ have appeared.

\section{A. A Discussion of the First Renormalization Transformation}

In this section we analyse operations necessary to calculate $T \rho_{0}$. With each positive term in the action $A(U)$ we connect a restriction on field variables $U$, which is a restriction on the corresponding plaquette variable. We introduce the decomposition of unity

$$
1=\sum_{P} \prod_{p \in P} \chi\left(\left\{|U(\partial p)-1| \geqq \varepsilon_{1}\right\}\right) \prod_{p \in \mathcal{P}^{\mathrm{C}}} \chi\left(\left\{|U(\partial p)-1|<\varepsilon_{1}\right\}\right),
$$

where the sum is over sets $P$ of plaquettes of the lattice $T$. We choose the number $\varepsilon_{1}$ in the same way as in the papers on the Higgs model, i.e. we take $\varepsilon_{1}=g_{0} p\left(g_{0}\right)$, where $p(g)=b_{0}\left(1+\log g^{-1}\right)^{p o}, p_{0}>2$ and $b_{0}$ is a sufficiently large absolute constant. Next we proceed as in [9], that is to each term of the decomposition we assign a subset $\Omega_{1} \subset T_{1}$ defined as a union of big blocks, i.e. blocks of the size $M_{1}$, of the unit lattice $T_{1}$, such that their distances to $P$ are $>R M_{1}$. We take $R=R_{1}\left(1+\log g_{0}^{-1}\right)^{r_{0}}=$ $R_{1} r\left(g_{0}\right)$. By this definition $p \subset \Omega_{1}^{c}$, in fact dist $\left(P, \Omega_{1}\right)>R M_{1}$, and plaquette variables of field configurations are small in a neighbourhood of $\Omega_{1}$. We can take a neighbourhood $\Omega_{0}$ such that $\Omega_{0} \backslash \Omega_{1}$ is a union of big blocks with distances to $\Omega_{1}$ smaller or equal to $R_{1} M_{1}$. Now we perform a partial resummation over all $P$ 
determining the same domain $\Omega_{1}$, and we write the decomposition (7) as

$$
1=\sum_{\text {admissible } \Omega_{1}} \zeta_{\Omega_{1}^{c}} \chi_{\Omega_{1}}
$$

where $\chi_{\Omega_{1}}$ involves the characteristic functions in (7) connected with plaquettes $p \in \Omega_{1}$, i.e. plaquettes with at least one corner belonging to $\Omega_{1}$. The meaning of $\zeta_{\Omega_{1}^{c}}$ is clear.

The underintegral expression in $T \rho_{0}$ is invariant with respect to gauge transformations $u$ fixed to 1 at points of the new lattice $T^{(1)}$, i.e. $u(y)=1$ for $y \in T^{(1)}$. We remove this freedom in the domain $\Omega_{1}$ by a simple Faddeev-Popov procedure, using the identity

$$
\begin{aligned}
& \prod_{y \in \Omega_{1}^{(1)}} \int_{x \in B(y), x \neq y} d u(x) \delta_{A x(y)}\left(U^{u}\right)=1, \\
& \delta_{A x(y)}(U)=\prod_{x \in B(y), x \neq y} \delta\left(U\left(\Gamma_{y, x}\right)\right) .
\end{aligned}
$$

We obtain the following equality

$$
\begin{aligned}
\left(T \rho_{0}\right)(V)= & \sum_{\Omega_{1}} \int d U \delta\left(\bar{U} V^{-1}\right) \delta_{A x\left(\Omega_{1}\right)}(U) \zeta_{\Omega_{1}^{c}} \chi_{\Omega_{1}} \\
& \times \exp \left[-\frac{1}{g_{0}^{2}} A(U)-E\right]
\end{aligned}
$$

where

$$
\delta_{A x\left(\Omega_{1}\right)}(U)=\prod_{y \in \Omega_{1}^{(1)}} \delta_{A x(y)}(U) .
$$

Let us consider a term in the sum on the right-hand side above. For each plaquette $p \in P$, where $P$ is one of the sets occurring in the definition of $\zeta_{\Omega_{1}^{\mathrm{c}}}$, we have

$$
\exp \left[-\frac{1}{g_{0}^{2}}[1-\operatorname{Re} \operatorname{tr} U(\partial p)]\right]=\exp \left[-\frac{1}{2 g_{0}^{2}}|U(\partial p)-1|^{2}\right] \leqq \exp \left(-\frac{1}{2} p^{2}\left(g_{0}\right)\right),
$$

and by the definition of $g_{0}=g \varepsilon^{1 / 2}$ the number on the right-hand side is smaller than any positive power of $\varepsilon$. This small factor controls the part of the integral over a neighbourhood of the plaquette $p$, for example a neighbourhood of the radius $R M_{1}$, as in [9]. Thus the subintegral over $\Omega_{1}^{c}$ is controlled by the small factors connected with $\zeta_{\Omega_{1}^{c}}$, and we have to consider the subintegral over $\Omega_{1}$. This subintegral we calculate by the saddle point method. At first we have to find critical points of the action $A(U)$ on the region of integration. If a configuration $U$ belongs to this region, then it satisfies the regularity conditions $|U(\partial p)-1|<g_{0} p\left(g_{0}\right)$ on the domain $\Omega_{0}$, and by Proposition 1 from [4] the configuration $V=\bar{U}$ satisfies the conditions $\left|V\left(\partial p^{\prime}\right)-1\right|<2 L^{2} g_{0} p\left(g_{0}\right)$ on $\Omega_{0}^{(1)}$. We fix a configuration $U=V_{0}$ on $\Omega_{1}^{c}$ and we look for critical points of the functional

$$
U \rightarrow A(U) \text { for } U: U=V_{0} \text { on } \Omega_{1}^{c}, \quad \bar{U}=V \quad \text { on } \Omega_{1}^{(1)},
$$

$U$ satisfies axial gauge conditions on $\Omega_{1}$. 
For $g_{0}$ sufficiently small $2 L^{2} g_{0} p\left(g_{0}\right)<a_{1}$, where $a_{1}$ is the constant in Theorem 1 [7], hence there exists the exactly one critical configuration $U_{1}$ in a space of regular configurations satisfying the conditions in (12). Such a space contains the region of integration, so there is at most one critical point in the region. We make a change of variables in the integral over $\Omega_{1}$ taking $U=U^{\prime} U_{1}$. The new variables $U^{\prime}$ are called fluctuation fields, and the minimal configuration $U_{1}$ is called a background field.

The configuration $U_{1}$ satisfies the regularity conditions $\left|U_{1}(\partial p)-1\right|$ $<2 B_{3} g_{0} p\left(g_{0}\right)$ on $\Omega_{1}$, hence $U_{1}, U^{\prime} U_{1}$ satisfy the assumptions of Lemma 1 [6] with $\alpha_{0}=2 L^{2} B_{3} g_{0} p\left(g_{0}\right), \quad \alpha_{1}=0$, and the lemma yields the bound $\left|V^{\prime}-1\right|$ $<8 \cdot 3^{2} L^{2} B_{3} g_{0} p\left(g_{0}\right)$ for $g_{0}$ sufficiently small. We enlarge the region of integration to all configurations $V^{\prime}=e^{i A^{\prime}}$ satisfying $\left|A^{\prime}\right|<16 \cdot 3^{2} L^{2} B_{3} g_{0} p\left(g_{0}\right)$ on $\Omega_{1}$. Thus we get the inequality

$$
\begin{aligned}
\left(T \rho_{0}\right)(V) \leqq & \sum_{\Omega_{1}} \chi_{1} \int d V_{0}\left\lceil\Omega_{\Omega_{1}^{c}} \prod_{b^{\prime} \in \Omega_{1}^{c}} \delta\left(\bar{V}_{0}\left(b^{\prime}\right) V^{-1}\left(b^{\prime}\right)\right) \zeta_{\Omega_{1}^{c}}\right. \\
& \times \int d U^{\prime} \uparrow_{\Omega_{1}} \delta\left(\left(\overline{U^{\prime} U_{1}}\right)\left(\overline{U_{1}}\right)^{-1}\right) \delta_{A x\left(\Omega_{1}\right)}\left(U^{\prime}\right) \chi^{\prime} \exp \left[-\frac{1}{g_{0}^{2}} A\left(U^{\prime} U_{1}\right)-E\right]
\end{aligned}
$$

where

$$
\begin{aligned}
& \chi_{1}=\prod_{p^{\prime} \in \Omega_{1}^{(1)}} \chi\left(\left\{\left|V\left(\partial p^{\prime}\right)-1\right|<2 L^{2} g_{0} p\left(g_{0}\right)\right\}\right), \\
& \chi^{\prime}=\prod_{b \in \Omega_{1}} \chi\left(\left\{\left|A^{\prime}(b)\right|<16 \cdot 3^{2} L^{2} B_{3} g_{0} p\left(g_{0}\right)\right\}\right),
\end{aligned}
$$

and where we have put $U^{\prime}=1, U_{1}=V_{0}$ on $\Omega_{1}^{c}$.

Now we write the integrals over $U^{\prime}$ in terms of the variables $A^{\prime}$. For the expressions in $\delta$-functions defining the renormalization transformation we have

$$
\left(\overline{U^{\prime} U_{1}}\right)\left(\overline{U_{1}}\right)^{-1}=\tilde{U}^{\prime}=\overline{\bar{U}}^{\prime}=\exp i Q\left(A^{\prime}\right),
$$

where the second equality follows from the definition (89) (or (63)) in [4], and the gauge fixing conditions. Properties of the function $Q\left(A^{\prime}\right)=Q\left(U_{1}, A^{\prime}\right)$ were described in Proposition 3 [4]. It is an analytic function of $A^{\prime}$ with the decomposition

$$
Q\left(A^{\prime}\right)=Q A^{\prime}+C\left(A^{\prime}\right)
$$

where $Q=Q\left(U_{1}\right)$ is the linear averaging operator defined by (124) [4], and $C\left(A^{\prime}\right)$ is an analytic function with an expansion beginning with a second order polynomial. The $\delta$-function in (13) can be written as

$$
\left.\prod_{c \in \Omega_{1}^{(1)}} \delta\left(\overline{\left(\bar{U}^{\prime} U_{1}\right.}\right)(c)\left(\bar{U}_{1}(c)\right)^{-1}\right)=\prod_{c \in \Omega_{1}^{(1)}} \frac{1}{\sigma_{0}} \delta\left(Q\left(A^{\prime}, c\right)\right),
$$

where the $\delta$-functions on the right are defined on the vector space $\mathfrak{g}$, and concentrated at the origin of this space. The constant $\sigma_{0}$ will be discussed later. To write the integrals (13) as Gaussian integrals with small and local interactions we have to change variables in order to linearize the functions $Q\left(A^{\prime}, c\right)$. It is possible to do it separately for each function $Q\left(A^{\prime}, c\right)$, so the whole transformation will have good locality properties. Thus we are looking for a function $\tilde{D}(A, b, c)$ satisfying the 
equation

$$
Q(A-\widetilde{D}(A, c), c)=(Q A)(c)-Q \widetilde{D}(A, c)+C(A-\widetilde{D}(A, c), c)=(Q A)(c),
$$

or

$$
Q \widetilde{D}(A, c)=C(A-\widetilde{D}(A, c), c)
$$

Let us denote by $b_{0}(c)$ the bond $b \in B(c)$ and contained in $c$ (let us recall that $B(c)$ is the set of bonds connecting the blocks $B\left(c_{-}\right), B\left(c_{+}\right)$, i.e. the set of bonds $b$ such that $\left.b_{-} \in B\left(c_{-}\right), b_{+} \in B\left(c_{+}\right)\right)$. We assume that $\widetilde{D}(A, b, c)=0$ for all $b \neq b_{0}(c)$. This assures the locality properties. We have analyzed the equations of the type (17) several times already, see Sect. $E$ in [6], Sect. $C$ in [7]. There exists a unique solution $\widetilde{D}$ of this equation for $A$ sufficiently small, and it is an analytic function of $A$ with a Taylor expansion beginning with second order terms. Denoting the terms by $\widetilde{D}^{(2)}(A)$, we have $Q \tilde{D}^{(2)}(A, c)=C^{(2)}(A, c)$, and inspecting the formula (124) [4] for $Q$ we see easily that $\widetilde{D}^{(2)}\left(A, b_{0}(c), c\right)$ can be expressed as a linear operator on $g$ acting on $C^{(2)}(A, c)$. More generally we can get a system of recursive equations for terms in the expansion of $\widetilde{D}$. The function $\widetilde{D}(A, c)$, similarly as $Q\left(A^{\prime}, c\right)$, depends on $A$ restricted to $B\left(c_{-}\right) \cup B\left(c_{+}\right)$. It is this locality property which simplifies analysis of interaction terms in the integrals.

To write the integrals (13) in terms of the variables $A^{\prime}$ we express the Haar measure $d U^{\prime}$ as $d U^{\prime}=\sigma\left(A^{\prime}\right) d A^{\prime}=\sigma_{0} \sigma / \sigma_{0}\left(A^{\prime}\right) d A^{\prime}, \sigma_{0}=\sigma(0)$, where $d A^{\prime}$ is the Lebesque measure on $\mathfrak{g}$, and $\sigma\left(A^{\prime}\right)$ is a density which can be calculated explicitly for all classical groups. For example for $\operatorname{SU}(2)$ we have $\sigma(A)=1 / 2 \pi^{2}(\sin |A| / A \mid)^{2}$, where $|A|=$ $\sum_{a=1}^{3}\left(A^{a}\right)^{2}$, and an element $A$ of the Lie algebra is represented as $A=\sum_{a=1}^{3} \sigma_{a} A^{a}, \sigma_{a}$ are the three Pauli matrices (generators of the Lie algebra). Generally $\sigma(A)$ is an analytic, positive, even function of $A$ in a neighbourhood of $0 \in \mathfrak{g}$, invariant with respect to the adjoint representation of the group $G$, i.e. with respect to the all orthogonal transformations $R(U), U \in G$. Thus we have

$$
\begin{aligned}
\left(T \rho_{0}\right)(V) \leqq & \sum_{\Omega_{1}} \chi_{1} \int d V_{0} \uparrow_{\Omega_{1}^{c}} \prod_{b^{\prime} \subset \Omega_{1}^{c}} \delta\left(\bar{V}_{0}\left(b^{\prime}\right) V^{-1}\left(b^{\prime}\right)\right) \zeta_{\Omega_{1}^{c}} \\
& \times \int d A \uparrow_{\Omega_{1}} \operatorname{det}\left(I-\frac{\delta}{\delta A} \tilde{D}(A)\right) \frac{\sigma}{\sigma_{0}}(A-\widetilde{D}(A)) \delta(Q A) \delta_{A x}(A) \chi \\
& \times \exp \left[-\frac{1}{g_{0}^{2}} A\left(\exp i(A-\tilde{D}(A)) U_{1}\right)-E+\log \sigma_{0} \mid \Omega_{1}^{*}\right]
\end{aligned}
$$

where $\chi=\Pi_{b \in \Omega_{1}} \chi\left(\left\{|A(b)|<g_{0} p^{2}\left(g_{0}\right)\right\}\right), g_{0}$ sufficiently small, and $\left|\Omega_{1}^{*}\right|$ denotes the number of bonds belonging to $\Omega_{1}$ minus the number of bonds in $\Omega_{1}^{(1)}$ and minus the number of bonds in the axial gauge fixing set. The function in the exponential, considered on the space of configurations $A$ restricted by the $\delta$-functions and the characteristic functions $\chi$, has a minimum at $A=0$. This implies that a linear term in its expansion vanishes. This expansion, with a special emphasis on linear and quadratic terms, was described in [7], Eq. (26). The linear term is determined by the function $J=D_{U_{1}}^{*} \operatorname{Im} U_{1}$, and the quadratic term is determined by an operator of the form $D_{U_{1}}^{*} D_{U_{1}}+$ (a small perturbation), where $D_{U}$ denotes a covariant derivative. We 
have

$$
\begin{aligned}
A\left(\exp i(A-\tilde{D}(A)) U_{1}\right)= & A\left(U_{1}\right)-\langle\widetilde{D}(A), J\rangle+\frac{1}{2}\left\langle A-\tilde{D}(A), \Delta\left(U_{1}\right)\right. \\
& \times(A-\widetilde{D}(A))\rangle+V_{0}(A-\widetilde{D}(A)) \\
= & A\left(U_{1}\right)-\left\langle\widetilde{D}^{(2)}(A), J\right\rangle+\frac{1}{2}\left\langle A, \Delta\left(U_{1}\right) A\right\rangle+\widetilde{V}_{0}(A),
\end{aligned}
$$

where $\widetilde{V}_{0}(A)$ is defined by the last equality. It is an analytic function of $A$ with an expansion beginning with third order terms, and it is a sum over plaquette variables of functions which are almost local in $A$ and $U_{1}$. Let us exponentiate the determinant and the function $\sigma / \sigma_{0}$ in (18). The determinant can be written as

$$
\exp \operatorname{Tr} \log \left(I-\frac{\delta}{\delta A} \tilde{D}(A)\right)=\exp \left[\sum_{c \in \Omega_{1}^{(1)}} \operatorname{tr} \log \left(1-\frac{\partial}{\partial A\left(b_{0}(c)\right)} \tilde{D}\left(A, b_{0}(c), c\right)\right)\right]
$$

in the exponential the sum is over almost local functions. Similarly for the function $\sigma / \sigma_{0}$,

$$
\frac{\sigma}{\sigma_{0}}(A-\tilde{D}(A))=\exp \left[\sum_{b \in \Omega_{1}}\left(\log \frac{\sigma}{\sigma_{0}}\right)(A(b)-\tilde{D}(A, c(b), b))\right] .
$$

Let us denote the terms in the exponentials in $(20),(21)$ by $v(A)$.

Finally we make the transformation $A \rightarrow g_{0} A$ in (18). Taking into account this transformation and the formulas (19)-(21), we obtain

$$
\begin{aligned}
\left(T \rho_{0}\right)(V) \leqq & \left.\sum_{\Omega_{1}} \chi_{1} \int d V_{0}\right|_{\Omega_{1}^{c}} \prod_{b^{\prime} \subset \Omega_{1}^{c}} \delta\left(\bar{V}_{0}\left(b^{\prime}\right) V^{-1}\left(b^{\prime}\right)\right) \zeta_{\Omega_{1}^{c}} \\
& \times \int d A\left\lceil\Omega _ { 1 } \delta ( Q A ) \delta _ { A x } ( A ) \chi \operatorname { e x p } \left[\vartheta\left(g_{0} A\right)-\frac{1}{g_{0}^{2}} A\left(U_{1}\right)+\left\langle\tilde{D}^{(2)}(A), J\right\rangle\right.\right. \\
& \left.-\frac{1}{2}\left\langle A, \Delta\left(U_{1}\right) A\right\rangle-\frac{1}{g_{0}^{2}} \tilde{V}_{0}\left(g_{0} A\right)-E+\log \sigma_{0}\left|\Omega_{1}^{*}\right|+d(\mathfrak{g}) \log g_{0}\left|\Omega_{1}^{*}\right|\right] \\
= & \sum_{\Omega_{1}} \chi_{1} \int d V_{0} \mid \Omega_{\Omega_{1}^{c}} \prod_{b^{\prime} \subset \Omega_{1}^{c}} \delta\left(\bar{V}_{0}\left(b^{\prime}\right) V^{-1}\left(b^{\prime}\right)\right) \zeta_{\Omega_{1}^{c}} \exp \left[-\frac{1}{g_{0}^{2}} A\left(U_{1}\right)\right. \\
& \left.+\log Z^{(0)}\left(\Omega_{1}, U_{1}\right)-E+\log \sigma_{0}\left|\Omega_{1}^{*}\right|+d(\mathrm{~g}) \log g_{0}\left|\Omega_{1}^{*}\right|\right] \\
& \times \int d \mu_{\mathrm{C}^{(0)}\left(\Omega_{1}, U_{1}\right)}(A) \chi \exp \left[v\left(g_{0} A\right)-\frac{1}{g_{0}^{2}} \tilde{V}\left(g_{0} A\right)\right],
\end{aligned}
$$

where $\chi=\Pi_{b \in \Omega_{1}} \chi\left(\left\{|A(b)|<p^{2}\left(g_{0}\right)\right\}\right)$, and $d(\mathfrak{g})$ denotes a dimension of the Lie algebra $\mathrm{g}$.

The last integral above has the form $\int \mu \chi \exp V$, where $d \mu$ is a Gaussian measure with a covariance having an exponential decay property (and many other properties, see Sect. $E$ in [5]), and $V$ is a sum of terms with good localization properties. This integral can be expressed naturally by an exponentiated cluster expansion, but we use here the major simplification coming from the fact that the model is superrenormalizable and the scaled coupling constant $g_{0}$ is proportional to a positive power of $\varepsilon, g_{0}=g \varepsilon^{1 / 2}$. Using the ideas and methods of $[11,12,8,9]$ we expand $v\left(g_{0} A\right)-1 / g_{0}^{2} \widetilde{V}\left(g_{0} A\right)$ up to the sixth order (or higher) in $g_{0}$, and we estimate 
the remainder by $O\left(g_{0}^{7} p^{18}\left(g_{0}\right)\right)\left|\Omega_{1}\right| \leqq O\left(\varepsilon^{3+\kappa_{0}}\right)\left|T_{1}\right|, \kappa_{0}>0$. Next, the integral is calculated by the cumulant expansion formula (3.24) [8], again up to the sixth order in $g_{0}$. A result of the calculation can be represented by lower order connected vacuum graphs with vertices determined by the expansions of the function $\vartheta\left(g_{0} A\right)$ $-1 / g_{0}^{2} \widetilde{V}\left(g_{0} A\right)$. The vertices are represented by sums over almost local expressions, i.e. expressions involving field variables $A$ localized to a union of several neighbouring blocks. They have at most eight legs, and the graphs have at most six vertices.

We analyze these perturbative expressions in a way similar to the analysis in [10]. We localize vertices in big blocks by a decomposition of unity, and we expand the propagators $C^{(0)}\left(\Omega_{1}, U_{1}\right)$ into the generalized random walk expansion described in [5], Theorem 3.15, Theorem 3.10 and the preceding theorems. This gives a sum of expressions having the following structure. Each expression corresponds to a graph with vertices localized in cubes $\left\{\square_{i}\right\}$. A line of the graph connecting vertices $\square_{i}, \square_{j}$ is replaced by a random walk $\omega=\left(\left(\alpha_{0}, X_{0}\right),\left(\alpha_{1}, X_{1}\right)_{1} \ldots,\left(\alpha_{n}, X_{n}\right)\right)$ satisfying $\square_{i} \cap X_{0} \neq \phi, X_{m-1} \cap X_{m} \neq \phi, m=1, \ldots, n, X_{m} \cap \square_{j} \neq \phi$. A term in the expression, corresponding to the walk $\omega$, satisfies the bound (3.108) [5], i.e. can be bounded by

$$
O(1) O\left(M_{1}^{-(1 / 2)}\right)^{|\omega|} M_{1}^{-(1 / 2)|\omega|} \exp \left(-\frac{1}{2} \delta_{0} d\left(\omega, \square_{i}, \square_{j}\right)\right) \text {, }
$$

where $d\left(\omega, \square_{i}, \square_{j}\right)$ is the length of a shortest tree graph passing through $\square_{i}, \square_{j}$, $\left\{X_{m}\right\}$. Let us recall that the sets $X_{m}$ are connected unions of several big blocks. The localizations $\left\{\square_{i}\right\}$ and the walks $\omega$ replacing lines of the graph define a localization $X$ of the considered expression. This localization is simply a union of all these sets. It is easy to see that, because of the bound (23), the expressions corresponding to big localization sets $X$ are very small, especially if $X$ is not contained in a cube of the size $R M_{1}$, then the exponential factor in (23) yields the factor $\exp (-R)$, which is smaller than arbitrary power of $\varepsilon$. We estimate all such expressions using this bound and we get $O\left(\varepsilon^{\kappa}\right)\left|T_{1}\right|$. Summing the expressions with the same localization $X$ we get finally the inequality

$$
\begin{array}{r}
\int d \mu_{C^{(0)}\left(\Omega_{1}, U_{1}\right)}(A) \chi \exp \left[\vartheta\left(g_{0} A\right)-\frac{1}{g_{0}^{2}} \tilde{V}\left(g_{0} A\right)\right] \\
\leqq \exp \left[\sum_{X} \mathscr{P}_{1}^{\prime}\left(g_{0}, X, U_{1}\right)+O\left(\varepsilon^{3+\kappa_{0}}\right)\left|T_{1}\right|\right],
\end{array}
$$

where the sum is over localizations $X$ which have diameter smaller than $R M_{1}$. Localizations $X$ are connected unions of big blocks. Following [19] we define a linear size $\mathscr{L}(X)$ of a localization $X$ as the length of a shortest tree graph connecting the centers of big blocks in $X$, and other points, if the big blocks are scaled to unit cubes, i.e. the distance between centers of neighbouring blocks is taken to be equal to 1. With this definition we have

$$
\left|\mathscr{P}_{1}^{\prime}\left(g_{0}, X, U_{1}\right)\right| \leqq O\left(g_{0}\right) e^{-\kappa \mathscr{L}(X)},
$$

where $\kappa$ can be arbitrarily large if $M_{1}$ is sufficiently large. Besides the bounds (25) the expressions $\mathscr{P}_{1}^{\prime}$ have three very important properties. The first is gauge invariance with respect to all gauge transformations of the configuration $U_{1}$, i.e. the following 
equalities hold

$$
\mathscr{P}_{1}^{\prime}\left(g_{0}, X, U_{1}^{u}\right)=\mathscr{P}_{1}^{\prime}\left(g_{0}, X, U_{1}\right),
$$

for all gauge transformations $u$. The second is a localization property with respect to $U_{1}$. The expression $\mathscr{P}_{1}^{\prime}\left(g_{0}, X, U_{1}\right)$ depends on $U_{1}$ restricted to the set $\tilde{X}^{5}$ (1et us recall that $\left.\tilde{X}^{5}=\cup_{\square \subset X} \tilde{\square}^{5}\right)$. The third property is the analyticity with respect to $U_{1}$. These properties follow from the results of previous papers; let us make a comment only on the gauge invariance (26). It follows from the invariance of the expressions in (13), if we make the simultaneous transformations $U_{1} \rightarrow U_{1}^{u}, A^{\prime} \rightarrow R(u) A^{\prime}$, we have to notice only that all the expansions and expressions introduced later preserve this property. The measure $d A$ is invariant with respect to the orthogonal transformations $A \rightarrow R(u) A$, hence the desired invariance (26) follows.

Now we are ready to perform the most important operation, a renormalization of the interaction terms $\mathscr{P}_{1}^{\prime}$. Let us consider a term $\mathscr{P}_{1}^{\prime}\left(g_{0}, X, U_{1}\right)$. The localization domain $X$ is contained in a cube $\square$ of the size $R M_{1}$. We may assume that $\tilde{X}^{5} \subset \square$ also, and a center of $\square$ belongs to $X$. We apply the constructions and results of Sect. $F[7]$. According to these there exists a gauge transformation in a neighbourhood of $\square_{1}$, where $\square_{1}$ is a cube of the size $3 R M_{1}$ and with the same center as $\square$, such that the gauge transformed $U_{1}$ is represented as $\exp i \mathscr{H}(B)$ in the neighbourhood of $\square_{1}$. The function $\mathscr{H}(B)$ is represented as $\mathscr{H}(B)=H B+A_{1}$, where $H B$ corresponds to a linearized theory, and $A_{1}$ is a non-linear perturbation determined by Eq. (158) [7]. Let us explain now the configuration $B$, which plays an important role in our considerations. Let $y$ denote the center of $\square$. The configuration $B$ restricted to $\square_{1}$ is defined on $\square_{1}^{(1)}=\square_{1} \cap T^{(1)}$ and for a bond $c$ of this set is given by

$$
B(c)=\frac{1}{i} \log V\left(\Gamma_{y, c_{-}} \cup c \cup \Gamma_{c_{+}, y}\right) \text {. }
$$

The characteristic functions $\chi_{1}$ defined in (13) give the restrictions $\left|V\left(\partial p^{\prime}\right)-1\right|<$ $2 L^{2} g_{0} p\left(g_{0}\right)$, hence

$$
|B(c)|<4 L^{2}\left|c_{-}-y\right| g_{0} p\left(g_{0}\right)<8 L^{2} 3 R_{1} M_{1} r\left(g_{0}\right) g_{0} p\left(g_{0}\right),
$$

and for $g_{0}$ sufficiently small the number on the right-hand side above is small. This bound, with a different absolute constant, extends to the whole configuration $B$, and this assures that the theorems of [7] are applicable in the present situation. The bound (28) implies a similar bound for $\mathscr{H}(B)$, with the additional constant $B_{3}$ on the right-hand side.

By the gauge invariance (26), we have

$$
\mathscr{P}_{1}^{\prime}\left(g_{0}, X, U_{1}\right)=\mathscr{P}_{1}^{\prime}\left(g_{0}, X, \exp i \mathscr{H}(B)\right)
$$

and we expand the function with respect to $\mathscr{H}(B)$. Because of the bound (28) it is enough to expand up to the sixth order, hence

$$
\begin{aligned}
\mathscr{P}_{1}^{\prime}\left(g_{0}, X, \exp \dot{H}(B)\right)= & \mathscr{P}_{1}^{\prime}\left(g_{0}, X, 1\right)+\left\langle\left(\frac{\delta}{\delta \mathscr{H}} \mathscr{P}_{1}^{\prime}\right)\left(g_{0}, X, 1\right), \mathscr{H}(B)\right\rangle \\
& +\sum_{n=2}^{6} \frac{1}{n !}\left\langle\left(\frac{\delta^{n}}{\delta \mathscr{H}^{n}} \mathscr{P}_{1}^{\prime}\right)\left(g_{0}, X, 1\right), \otimes^{n} \mathscr{H}(B)\right\rangle+O\left(g_{0}^{7}\right) e^{-\kappa \mathscr{P}(X)}
\end{aligned}
$$


The gauge invariance (26) implies the invariance with respect to the global transformations $R(U), U \in G$, hence the equality

$$
R(U)\left(\frac{\delta}{\delta \mathscr{H}(b)} \mathscr{P}_{1}^{\prime}\right)\left(g_{0}, X, 1\right)=\left(\frac{\delta}{\delta \mathscr{H}(b)} \mathscr{P}_{1}^{\prime}\right)\left(g_{0}, X, 1\right)
$$

We have to notice only that (26) holds for all regular gauge field configurations, not only for the minimal configurations $U_{1}$. The derivative in the above formula is an element of the Lie algebra $\mathfrak{g}$, and by the assumption that $\mathfrak{g}$ is semi-simple, the only element invariant is 0 , and we conclude

$$
\left(\frac{\delta}{\delta \mathscr{H}(b)} \mathscr{P}_{1}^{\prime}\right)\left(g_{0}, X, 1\right)=0 .
$$

It is the only place we use the semi-simplicity, but the above conclusion is a fundamental point in our method. In the renormalization group language it is the statement that there are no relevant variables in the effective action.

Let us consider the terms in the sum over $n$ in (30). The function $\mathscr{H}(B)=H B+A_{1}$ is determined by the solution $A_{1}$ of Eq. (158) [7], and this solution is an analytic function of $H B$, or $B$. We expand it up to the sixth order at most, and we estimate remainders by the last term in (30). Finally we estimate terms with $B$ localized in $\square_{1}^{c}$ by the last term in (30) with the constant proportional to an arbitrary power of $g_{0}$. It is possible because the uniform exponential decay of all propagators provides the exponential factor $\exp \left(-1 / 2 \delta_{0} \operatorname{dist}\left(X, \square_{1}^{c}\right)\right) \leqq \exp \left(-R_{1} r\left(g_{0}\right)\right)$ in this case. Thus we obtain expressions which are polynomials in $B$ of at least the second order, and at most the sixth order, localized in $\square_{1}$. For each variable $B(c)$ there is a sequence of propagators connecting the bond $c$ with the set $X$. They provide the exponential factor $\exp \left(-\delta_{0} \operatorname{dist}(c, X)\right)$. This together with the exponential factor on the righthand side of (25) give the factor $\exp \left(-\kappa_{1} M_{1}^{-1}\left|c_{-}-y\right|\right)$, with $\kappa_{1}=1 / 7 \kappa$. We use the remaining $\exp \left(-\kappa_{1} \mathscr{L}(X)\right)$ to control a sum over all $X$ contributing to a given monomial in variables $B$.

Thus we obtain the following very simple representation

$$
\sum_{X} \mathscr{P}_{1}^{\prime}\left(g_{0}, X, U_{1}\right)=\sum_{X} \mathscr{P}_{1}^{\prime}\left(g_{0}, X, 1\right)+\sum_{Y} \mathscr{P}_{1}\left(g_{0}, Y, U_{1}\right)+O\left(\varepsilon^{3+\kappa_{0}}\right)\left|T_{1}\right|,
$$

where $Y=\left(y, c_{1}, \ldots, c_{n}\right), y$ represents a big block, i.e. $y \in \Omega_{1} \cap M_{1} Z^{3}, c_{i}$ are bonds in $\Omega_{1}^{(1)},\left|c_{i,-}-y\right|<R M_{1}$,

$$
\begin{aligned}
\mathscr{P}_{1}\left(g_{0}, Y, U_{1}\right) & =\left\langle\mathscr{P}_{1}\left(g_{0}, Y\right), B_{1}\left(c_{1}\right), \ldots, B_{1}\left(c_{n}\right)\right\rangle, \quad n \geqq 2, \\
B_{1}(c) & =\frac{1}{i} \log \bar{U}_{1} \Gamma_{y, c_{-}} \cup c \cup \Gamma_{c_{+}, y}, \\
\left|\mathscr{P}_{1}\left(g_{0}, Y\right)\right| & \leqq O\left(g_{0}\right) \prod_{i=1}^{n} \exp \left(-\kappa_{1} M_{1}^{-1}\left|c_{i,-}-y\right|\right) .
\end{aligned}
$$

The above representation is very convenient to work with, because the expressions $\mathscr{P}_{1}\left(g_{0}, Y, U_{1}\right)$ behave like irrelevant variables as they are at least second power in loop variables $B_{1}$.

Let us consider now the first sum on the right-hand side of (33). Of course it is a 
part of the vacuum energy renormalization counterterm. To obtain the whole counterterm we have to add a sum of the corresponding terms with localizations $X$ satisfying $X \cap \Omega_{1}^{c} \neq \phi$. This sum can be bounded by $O\left(g_{0}\right)\left|\Omega_{1}^{c}\right|$. We may define the counterterm either in the form of the unrestricted sum, or we may perform operations inverse to those done up to now and to replace the sum by the usual perturbative expression on the whole lattice $T_{1}$, with the background field $U_{1}$ replaced by 1 . We have to supplement also the constants in (22), involving $\log \sigma_{0}$ and $\log g_{0}$, to the whole lattice. Finally we normalize $\log Z^{(0)}\left(\Omega_{1}, U_{1}\right)$ subtracting and adding the term $\log Z^{(0)}\left(\Omega_{1}, 1\right)$, which we have to supplement to the whole lattice using the bound

$$
\left|\log \frac{Z^{(0)}\left(\Omega_{1}, 1\right)}{Z^{(0)}\left(T_{1}, 1\right)}\right| \leqq O(1)\left|\Omega_{1}^{c}\right| .
$$

The term $\log Z^{(0)}\left(\Omega_{1}, U_{1}\right)-\log Z^{(0)}\left(\Omega_{1}, 1\right)$ can be decomposed in a similar way as the integral in (24), and we get an expression $\Sigma_{Y} \mathscr{P}_{1}\left(Y, U_{1}\right)$ which is identical to the expression on the right-hand side of (33), only the coefficients do not depend on $g_{0}$. This expansion we analyse in the general case later.

Gathering together the inequalities and transformations we obtain finally

$$
\begin{aligned}
\left(T \rho_{0}\right)(V)= & \rho_{1}(V) \leqq \\
\times \exp [ & -\left.\frac{1}{\Omega_{1}} \chi_{1} \int d V_{0}\right|_{\Omega_{1}^{c}} \delta\left(\bar{V}_{0} V^{-1}\left(U_{1}\right)+\sum_{Y} \mathscr{P}_{1}\left(g_{0}, Y, U_{1}\right)\right. \\
& \left.\quad-E_{1}+O\left(\log g_{0}^{-1}\right)\left|\Omega_{1}^{c}\right|+O\left(\varepsilon^{3+\kappa_{0}}\right)\left|T_{1}\right|\right],
\end{aligned}
$$

where $g_{1}=g(L \varepsilon)^{1 / 2}, E_{1}=E-E^{(0)}$, and

$$
E^{(0)}=\log \sigma_{0}\left|T_{1}^{*}\right|+d(\mathrm{~g}) \log g_{0}\left|T_{1}^{*}\right|+\log Z^{(0)}\left(T_{1}, 1\right)+\sum_{X} \mathscr{P}_{1}^{\prime}\left(g_{0}, X, 1\right) .
$$

This inequality is the basis of our inductive assumption in the next section. In the proof we will use also many transformations and estimates discussed above, so we will not need to repeat them.

Let us make a remark about a lower bound. We introduce restrictions on fields in a slightly different way. The characteristic function $\chi_{1}$ denotes restrictions on $V$ of the form $\left|U_{1}(\partial p)-1\right|<L^{-2} g_{0} p\left(g_{1}\right)$, where $U_{1}=U_{1}(V)$ is the minimal configuration determined by $V$. The restrictions on $A$ are introduced as in (18), and we perform the same operations on the whole lattice as on the sets $\Omega_{1}$. They give the inequality

$$
\rho_{1}(V) \geqq \chi_{1} \exp \left[-\frac{1}{g_{1}^{2}} A^{L^{-1}}\left(U_{1}\right)+\sum_{Y} \mathscr{P}_{1}\left(g_{0}, Y, U_{1}\right)-E_{1}-O\left(\varepsilon^{3+\kappa_{0}}\right)\left|T_{1}\right|\right] .
$$

\section{B. An Inductive Assumption on the Form of Approximate Effective Actions}

In this section we formulate inequalities satisfied by all the actions $\rho_{k}, k=1,2, \ldots, K$. To write them we have to introduce some new definitions and notations. At first let 
us explain a geometric setting. In the first step we have defined the small fields regions $\Omega_{1}$. In the next step we introduce a decomposition of unity (7), but on the domain $\Omega_{1}^{(1)}$ only and with $\varepsilon_{1}=g_{1} p\left(g_{1}\right)$. Each term in the decomposition determines a small fields region $\Omega_{2}$ in almost the same way as in the first step, the only difference is that we take blocks with distances to $P \cup \Omega_{1}^{c}$ greater than $R M_{1}$, where $R=R_{1} r\left(g_{1}\right)$. Thus we obtain a sequence of domains

$$
\Omega_{1} \supset \Omega_{2} \supset \cdots \supset \Omega_{k}, \quad \Omega_{j} \subset T_{n},
$$

satisfying the conditions

$$
\left(L^{j} \eta\right)^{-1} \operatorname{dist}\left(\Omega_{j}^{c}, \Omega_{j+1}\right)>R\left(g_{j}\right) M_{1}, \quad R\left(g_{j}\right)=R_{1} r\left(g_{j}\right),
$$

$\Omega_{j}$ is a union of big blocks of the size $M_{1} L^{j} \eta$.

We define the sets $\Lambda_{j}, \mathfrak{B}$ as in [5], i.e. $\Lambda_{j}=\Omega_{j}^{(j)} \backslash \Omega_{j+1}^{(j)}$, and we denote $Z_{j}=\Omega_{j+1}^{(j) c}$ $\subset T_{L_{\eta} \eta_{\eta}}^{(j)}$. Gauge field configurations $V_{j}$ are defined on $Z_{j}, j=0,1, \ldots, k-1$, and functions $\zeta_{A_{j}}$ are defined in the same way as $\zeta_{\Omega_{1}^{c}}$ on configurations $V_{j}$ restricted to $\Lambda_{j}$. Finally characteristic functions $\chi_{j}$ are generalizations of the characteristic function $\chi_{1}$ and are defined by

$$
\chi_{j}=\prod_{p \in A_{j}} \chi\left(\left\{\left|V_{j}(\partial p)-1\right|<2 L^{2} g_{j-1} p\left(g_{j-1}\right)\right\}\right), \quad j=1, \ldots, k,
$$

where we have denoted $V_{k}=V$.

Our inductive assumption has the following form

$$
\begin{aligned}
\rho_{k}(V) \leqq & \sum_{\left\{\Omega_{j}\right\}} \int d V_{k-1}\left\{Z_{k-1} \delta\left(\bar{V}_{k-1} V^{-1}\right) \cdots\right. \\
& \times \int d V_{0} \mid z_{0} \delta\left(\bar{V}_{0} V_{1}^{-1}\right) \chi_{k} \zeta_{A_{k-1}} \chi_{k-1} \cdots \zeta_{A_{1}} \chi_{1} \zeta_{\Omega_{1}^{c}} \\
& \times \exp \left[-\frac{1}{g_{k}^{2}} A^{\eta}\left(U_{k}\right)+\sum_{j=1}^{k} \sum_{Y_{j}} \mathscr{P}_{j}\left(Y_{j}, U_{k}\right)-E_{k}\right. \\
& \left.+\sum_{j=0}^{k-1} O\left(\log g_{j}^{-1}\right)\left|Z_{j}\right|+\sum_{j=0}^{k-1} O\left(\left(L^{j} \varepsilon\right)^{3+\kappa_{0}}\right)\left|T_{1}^{(j)}\right|\right] .
\end{aligned}
$$

Here the sets $Z_{j}$ are rescaled to the unit scale. The configuration $U_{k}$ is determined by the variational problem considered in [7], i.e. it is a minimum of the functional

$$
\begin{aligned}
& U \rightarrow A^{\eta}(U), \quad U: \bar{U}^{j}=V_{j} \text { on } A_{j}, \quad j=0,1, \ldots, k, \\
& \text { where we have put } \Lambda_{0}=\Omega_{1}^{c} \text { and } V_{k}=V .
\end{aligned}
$$

A definition of $E_{\mathrm{k}}$ is clear from (36), and will be discussed later also. The expressions $\mathscr{P}_{j}\left(Y_{j}, U_{k}\right)$ are defined similarly to $(35): Y_{j}=\left(y, c_{1}, \ldots, c_{n}\right)$, where $y$ represents big blocks of $L_{\eta}^{j}$-lattice, contained in $\Omega_{k}$, i.e. $y \in \Omega_{k}^{(j)} \cap M_{1} L^{j} \eta Z^{3}$, and $c_{i}$ are bonds in $\Omega_{k}^{(j)}$, $\left|c_{i,-}-y\right|<R\left(g_{j}\right) M_{1} L^{j} \eta$

$$
\begin{aligned}
\mathscr{P}_{j}\left(Y_{j}, U_{k}\right) & =\left\langle\mathscr{P}_{j}\left(Y_{j}\right), \quad B_{k}\left(c_{1}\right), \ldots, B_{k}\left(c_{n}\right)\right\rangle, \quad n \geqq 2, \\
B_{k}(c) & =\frac{1}{i} \log \bar{U}_{k}^{j}\left(\Gamma_{y, c_{-}} \cup \mathcal{C} \cup \Gamma_{c_{+}, y}\right), \quad c \in \Omega_{k}^{(j)}, \\
\left|\mathscr{P}_{j}\left(Y_{j}\right)\right| & \leqq O(1) \prod_{i=1}^{n} \exp \left(-\kappa_{1}\left(M_{1} L^{j} \eta\right)^{-1}\left|c_{i,-}-y\right|\right) .
\end{aligned}
$$


The configuration $U_{k}$ satisfies the following regularity condition on $\Omega_{k}: \mid U_{k}(\partial p)$ $-1 \mid<2 L^{2} B_{3} g_{k-1} p\left(g_{k-1}\right) \eta^{2}$. This implies the condition $\left|\bar{U}_{k}^{j}\left(\partial p^{\prime}\right)-1\right|$ $<4 L^{2} B_{3} g_{k-1} p\left(g_{k-1}\right)\left(L^{j} \eta\right)^{2}$ for $p^{\prime} \subset \Omega_{k}^{(j)}$, and from (43) we get

$$
\begin{aligned}
\left|\mathscr{P}_{j}\left(Y_{j}, U_{k}\right)\right| \leqq & O(1) \prod_{i=1}^{n} \exp \left(-\kappa_{1}\left(M_{1} L^{j} \eta\right)^{-1}\left|c_{i,-}-y\right|\right) \\
& \times\left(L^{j} \eta\right)^{-1}\left|c_{i,-}-y\right| 8 L^{2} B_{3} g_{k-1} p\left(g_{k-1}\right)\left(L^{j} \eta\right)^{2} .
\end{aligned}
$$

By the assumption $n \geqq 2$, summation over all $Y_{j}$ with $y$ fixed yields for $g_{k-1}$ sufficiently small

$$
\sum_{Y_{j} y=y_{0}}\left|\mathscr{P}_{j}\left(Y_{j}, U_{k}\right)\right| \leqq O(1)\left(O\left(M_{1}^{3}\right) g_{k-1} p\left(g_{k-1}\right)\right)^{2}\left(L^{j} \eta\right)^{4} .
$$

Summation over $y$ gives the factor $\left(M_{1} L^{j} \eta\right)^{-3}\left|\Lambda_{k}\right|$, and finally summation over $j=1, \ldots, k$ gives the following bound for the sum of interaction terms in (41),

$$
\sum_{j=1}^{k} \sum_{Y_{j}}\left|\mathscr{P}_{j}\left(Y_{j}, U_{k}\right)\right| \leqq O(1) M_{1}^{3} g_{k-1}^{2} p^{2}\left(g_{k-1}\right)\left|\Lambda_{k}\right| .
$$

Thus the sum is not only convergent, but also small. Of course the condition $n \geqq 2$ plays a crucial role in the above bounds. We will use them in several other important points.

Analogously to (37) we assume the lower bound

$$
\rho_{k}(V) \geqq \chi_{k} \exp \left[-\frac{1}{g_{k}^{2}} A^{\eta}\left(U_{k}\right)+\sum_{j=1}^{k} \sum_{Y_{j}} \mathscr{P}_{j}\left(Y_{j}, U_{k}\right)-E_{k}-\sum_{j=0}^{k-1} O\left(\left(L^{j} \varepsilon\right)^{3+\kappa_{0}}\right)\left|T_{1}^{(j)}\right|\right],
$$

where the characteristic function $\chi_{k}$ corresponds to the restrictions on $V$ given by the conditions $\left|U_{k}(\partial p)-1\right|<g_{k} p\left(g_{k}\right) \eta^{2}, p \subset T_{n}$.

Let us make an additional comment concerning the bound (46). It is implied by the bound (44) for the functions $\mathscr{P}_{j}\left(Y_{j}, U_{k}\right)$. Each renormalization transformation increases the index of the minimal configuration by 1 , hence the right-hand side of the bound (44) decreases by the factor $L^{-2 n}$. Because $n \geqq 2$, so $L^{-2 n} \leqq L^{-4}$ and the functions $\mathscr{P}_{j}\left(Y_{j}, U_{k}\right)$ behave like irrelevant variables in the dimensions 3 , although in bounds only, not in exact scaling properties. For the Wilson formulation of lattice gauge theories, with group valued gauge field configurations, we do not have scaling transformations. In the four-dimensional case the functions $\mathscr{P}_{j}\left(Y_{j}, U_{k}\right)$ with $n=2$ behave like marginal variables, and an additional renormalization, a coupling constant renormalization, is needed.

\section{Renormalization Transformation Preserves The Form of the Inductive Inequality}

We apply the renormalization transformation $T$ to the density $\rho_{k}$, and we use the inductive inequality (41). Thus the density $\rho_{k+1}$ is bounded by a sum of terms obtained by application of the renormalization transformation $T$ to terms on the right-hand side of (41). Now we do the same operations as in the first step. We introduce the decomposition of unity (7) for the field $V$ on the domain $\Lambda_{k}$, with $\varepsilon_{1}=g_{k} p\left(g_{k}\right)$. Let us denote the field variables $V$ by $V_{k}$, and the new fields by $V$. We 
define the set $\Omega_{k+1}^{(k)}$ as a union of big blocks of the lattice $T_{1}^{(k)}$, with distances to $P \cup \Omega_{k}^{(k) c}$ greater than $R\left(g_{k}\right) M_{1}$. We change the definition of $\Lambda_{k}$, taking $\Lambda_{k}$ $=\Omega_{k}^{(k)} \backslash \Omega_{k+1}^{(k)}$, and we define $\Lambda_{k+1}=\Omega_{k+1}^{(k+1)}$, hence $\Omega_{k+1}^{(k)}=B\left(\Lambda_{k+1}\right)$. The partial resummation over admissible $P$ gives the function $\zeta_{A_{k}}$ defined on fields $V_{k}$ restricted to $\Lambda_{k}$, and the small fields characteristic function on a neighbourhood of $B\left(\Lambda_{k+1}\right)$ allows us to introduce the functions $\chi_{k+1}$ given by (41), with $V$ instead of $V_{k+1}$. We decompose the integral over $V_{k}$ into two parts. The first is simply the integral restricted to $Z_{k}=B\left(\Lambda_{k+1}\right)^{c}$, hence it defines the first expression

$$
\int d V_{k} \uparrow_{Z_{k}} \delta\left(\bar{V}_{k} V^{-1}\right) \cdots
$$

in the inductive formula for $k+1$. The second gives the integral

$$
\chi_{k-1} \int d V_{k} \uparrow_{B\left(\Lambda_{k+1}\right)} \delta\left(\bar{V}_{k} V^{-1}\right) \chi \exp \left[-\frac{1}{g_{k}^{2}} A^{\eta}\left(U_{k}\right)+\cdots\right],
$$

which we have to calculate. The symbol $\chi$ denotes characteristics functions in the decomposition of unity, restricted to $B\left(\Lambda_{k+1}\right)$, i.e. determining the small fields restrictions. The integral (49) is calculated by the saddle point method. We take the minimal configuration $V_{k}^{(k)}$ of the functional $A^{\eta}\left(U_{k}\right)$, which satisfies the conditions $V_{k}^{(k)} \uparrow_{z_{k}}=V_{k} \uparrow_{z_{k}}, \overline{V_{k}^{(k)}}=V$ on $\Lambda_{k+1}$. If we substitute it in $U_{k}$ in place of $V_{k} \uparrow_{A_{k}}$, we get the configuration $U_{k+1}$. We introduce the gauge fixing terms $\delta_{A x\left(B\left(\Lambda_{k+1}\right)\right)}\left(V_{k}\right)$ using $(9)$, and we make the translation $V_{k}=V_{k}^{\prime} V_{k}^{(k)}$. The restrictions on $V_{k}$ and $V_{k}^{(k)}$ imply that the configuration $V_{k}^{\prime}-1$ is small, more exactly $\left|V_{k}^{\prime}-1\right|<16 \cdot 3^{2} L^{2} B_{3} g_{k} p\left(g_{k}\right)$. The integration is expressed now in terms of the variables $V_{k}^{\prime}=e^{i A^{\prime}}$. We enlarge the region of integration to all configurations $V_{k}^{\prime}$ satisfying $\left|A^{\prime}\right|<32 \cdot 3^{2} L^{2} B_{3} g_{k} p\left(g_{k}\right)$, and we denote by $\chi^{\prime}$ the characteristic function of this region.

For the expressions in $\delta$-functions in (49) we have the formula analogous to (14),

$$
\left(\overline{V_{k}^{\prime} V_{k}^{(k)}}\right)\left(\overline{V_{k}^{(k)}}\right)^{-1}=\bar{V}_{k}^{\prime}=\exp i Q\left(A^{\prime}\right),
$$

the only difference is that the configuration $U_{1}$ is replaced by $V_{k}^{(k)}$, which has the same properties as $U_{1}$. Next we do the same operations as in the first step, and we obtain the inequality

$$
\begin{aligned}
& \text { (The integral }(49)) \leqq \chi_{k+1} \int d A_{\mid B\left(\Lambda_{k+1}\right)} \operatorname{det}\left(I-\frac{\delta}{\delta A} \tilde{D}(A)\right) \times \frac{\sigma}{\sigma_{0}}(A-\tilde{D}(A)) \delta(Q A) \delta_{A x}(A) \chi \\
& \quad \times \exp \left[-\frac{1}{g_{k}^{2}} A^{\eta}\left(U_{k}\left(\exp i(A-\widetilde{D}(A)) V_{k}^{(k)}\right)\right)+\cdots+\log \sigma_{0}\left|B\left(\Lambda_{k+1}\right)^{*}\right|\right]
\end{aligned}
$$

where $\chi=\Pi_{b \in B\left(\Lambda_{k+1}\right)} \chi\left(\left\{|A(b)|<g_{k} p^{2}\left(g_{k}\right)\right\}\right), g_{k}$ sufficiently small, $\left|B\left(\Lambda_{k+1}\right)^{*}\right|$ has the same meaning as $\left|\Omega_{1}^{*}\right|$ in the first step. Now we expand the action in $A$. Using the results of Sect. G [7] we write

$$
\begin{aligned}
& U_{k}\left(\exp i(A-\tilde{D}(A)) V_{k}^{(k)}\right)=\exp i \eta \mathscr{H}(A-\tilde{D}(A)) U_{k+1} \\
& \text { (modulo a gauge transformation), }
\end{aligned}
$$

where $\mathscr{H}(B)$ is determined by Eqs. (174),(175) [7]. Next using the gauge invariance of 
the action, the expansion (26), Eq. (174), and the formulas (74), (78)-(81) of [7], we get

$$
\begin{aligned}
A^{\eta}\left(U_{k}\left(\exp i(A-\widetilde{D}(A)) V_{k}^{(k)}\right)\right)=A^{\eta}\left(U_{k+1}\right)+\langle\mathscr{H}, J\rangle+\frac{1}{2}\left\langle\mathscr{H}, \Delta\left(U_{k+1}\right) \mathscr{H}\right\rangle+V_{0}(\mathscr{H}) \\
=A^{\eta}\left(U_{k+1}\right)+\left\langle H_{1}(A-\tilde{D}(A))+\mathscr{H}_{1}, J\right\rangle+\frac{1}{2}\left\langle H_{1}(A-\tilde{D}(A))+\mathscr{H}_{1},\right. \\
\left.\Delta_{1}\left(H_{1}(A-\tilde{D}(A))+\mathscr{H}_{1}\right)\right\rangle+V\left(H_{1}(A-\tilde{D}(A))+\mathscr{H}_{1}\right) \\
=A^{\eta}\left(U_{k+1}\right)+\frac{1}{2}\left\langle H_{1} A, \Delta_{1} H_{1} A\right\rangle-\left\langle H_{1} \tilde{D}^{(2)}(A), J\right\rangle+\left\{-\left\langle H_{1} \widetilde{D}_{3}(A), J\right\rangle\right. \\
\quad-\left\langle H_{1} A, \Delta_{1} H_{1} \tilde{D}(A)\right\rangle+\frac{1}{2}\left\langle H_{1} \widetilde{D}(A), \Delta_{1} H_{1} \widetilde{D}(A)\right\rangle \\
\left.\quad+\frac{1}{2}\left\langle\mathscr{H}_{1}, \Delta_{1} \mathscr{H}_{1}\right\rangle+V\left(H_{1} A-H_{1} \tilde{D}(A)+\mathscr{H}_{1}\right)\right\} .
\end{aligned}
$$

We have used also orthogonality relations following from the definitions of operations and configurations used above. For the quadratic form above we have

$$
\frac{1}{2}\left\langle H_{1} A, \Delta_{1} H_{1} A\right\rangle-\left\langle H_{1} \widetilde{D}^{(2)}(A), J\right\rangle=\frac{1}{2}\left\langle A, \Delta_{k} A\right\rangle,
$$

where $A_{k}$ was defined by (3.156) in [5], and investigated in Sect. E of that paper. Denoting the expression in curly brackets $\{\cdots\}$ in $(53)$ by $\widetilde{V}(A)$, using the definition (3.155) [5], the equalities (20), (21), the definition of $v(A)$, and doing the transformation $A \rightarrow g_{k} A$ in the integral, we obtain

(The integral (49)) $\leqq \chi_{k+1} \exp \left[-\frac{1}{g_{k}^{2}} A^{\eta}\left(U_{k+1}\right)-E_{k}+\log \sigma_{0}\left|B\left(\Lambda_{k+1}\right)^{*}\right|\right.$

$$
\begin{aligned}
& \left.+d(\mathfrak{g}) \log g_{k}\left|B\left(\Lambda_{k+1}\right)^{*}\right|+\log Z^{(k)}\left(B\left(\Lambda_{k+1}\right), U_{k+1}\right)\right] \\
& \times \int d \mu_{C^{(k)}\left(B\left(\Lambda_{k+1}\right), U_{k+1}\right)}(A) \chi \exp \left[v\left(g_{k} A\right)-\frac{1}{g_{k}^{2}} \tilde{V}\left(g_{k} A\right)\right. \\
& +\sum_{j=1}^{k} \sum_{Y_{j}} \mathscr{P}_{j}\left(Y_{j}, \exp \text { i } \mathscr{H}\left(g_{k} A-\tilde{D}\left(g_{k} A\right)\right) U_{k+1}\right) \\
& +(\text { the constants in (41)) }] .
\end{aligned}
$$

Now we repeat again the operations of the first step. We expand the functions in the last exponential above up to the sixth order in $g_{k}$, and we estimate the remainder. Let us discuss at first the expression $v\left(g_{k} A\right)-1 / g_{k}^{2} \tilde{V}\left(g_{k} A\right)$. The expansion of $\vartheta\left(g_{k} A\right)$ is exactly the same as in the first step. To expand $1 / g_{k}^{2} \tilde{V}\left(g_{k} A\right)$, we use the properties and expansions of the functions $V, \mathscr{H}, \mathscr{H}_{1}$, discussed in [7]. The remainders for both expressions are estimated by $O\left(g^{7} p^{18}\left(g_{k}\right)\right)\left|B\left(\Lambda_{k+1}\right)\right| \leqq O\left(\left(L^{k} \varepsilon\right)^{3+\kappa_{0}}\right)\left|T_{1}^{(k)}\right|$. Lower order terms are given by local polynomials for $v\left(g_{k} A\right)$, and by non-local polynomials for $1 / g 2^{k} \tilde{V} g_{k} A$ ). They correspond to tree graphs with vertices defined by terms in the expansions of local functions $V_{0}\left(A^{\prime}\right), C\left(A^{\prime}\right)\left(A^{\prime}\right.$ is a gauge field configuration on the $\eta$ lattice), and lines defined by propagators $H, H_{1}, \boldsymbol{G}$. External legs correspond to $H_{1} A$. An expression corresponding to such a graph has the form

$$
\sum_{b_{1}, \ldots, b_{m} \in B\left(A_{k+1}\right)}\left\langle v\left(g_{k}, b_{1}, \ldots, b_{m}\right), A\left(b_{1}\right), \ldots, A\left(b_{m}\right)\right\rangle, \quad m \geqq 3,
$$

and

$$
\left|v\left(g_{k}, b_{1}, \ldots, b_{m}\right)\right| \leqq O\left(g_{k}^{m-2}\right) \exp \left(-\frac{1}{2} \delta_{0} \mathscr{L}\left(\left\{b_{i}\right\}\right)\right)
$$


(let us recall that $\mathscr{L}\left(\left\{b_{i}\right\}\right)$ denotes the length of a shortest graph on the unit lattice, connecting points of $b_{i}$ and possibly other points).

Let us consider now expansions of the terms $\mathscr{P}_{j}$. We use the bounds (44) which hold not only for $U_{k}$, but for an arbitrary configuration having the same properties and bounds as $U_{k}$. We expand a term of this type in (55) with respect to $\mathscr{H}$ up to the sixth order; the remainder can be estimated by the right-hand side of (44), with an additional power of $g_{k} p\left(g_{k}\right)$ missing, so that the overall factor is $\left(g_{k} p\left(g_{k}\right)\right)^{7}$. Estimating next in a similar way as in (45), (46) we get $O\left(\left(L^{k} \varepsilon\right)^{3+\kappa_{0}}\right)\left|B\left(\Lambda_{k+1}\right)\right|$. For lower order terms we expand the function into powers of $g_{k} A$, and we estimate terms with an overall power greater than six as above. Lower order terms have again the form of non-local polynomials (56), but now a coefficient $v$ connected with the expansion of $\mathscr{P}_{j}\left(Y_{j}, U_{k}\right)$ has the estimate

$$
\begin{aligned}
\left|v\left(g_{k}, b_{1}, \ldots, b_{m}\right)\right| \leqq & O\left(g_{k}^{m}\right)\left\{\prod_{i=1}^{n} O(1) \exp \left(-\frac{1}{2} \kappa_{1}\left(M_{1} L^{j} \eta\right)^{-1}\left|c_{i,-}-y\right|\right)\right. \\
& \left.\times\left(L^{j} \eta\right)^{-1}\left|c_{i,-}-y\right|\left(L^{j} \eta\right)^{2}\right\} \exp \left(-\frac{1}{2} \delta_{0} \mathscr{L}\left(\left\{b_{i}\right\} \cup\{y\}\right)\right) .
\end{aligned}
$$

We sum up all the coefficients at the same monomial in $A$. We get a coefficient which, by bound similar to (45), (46), can be estimated as in (56) (with $O\left(g_{k}^{m}\right)$ ).

Thus these expansions and resummations give a non-local polynomial $\mathscr{V}(A)$, whose terms are described by (56). The integral on the right-hand side of (55) is estimated by

$$
\begin{aligned}
\exp \left[\sum_{j=1}^{k} \sum_{Y_{j}} \mathscr{P}_{j}\left(Y_{j}, U_{k+1}\right)+(\text { the constants in }(41))\right] \\
\times \int d \mu_{C^{(k)}}(A) \chi \exp \left[\mathscr{V}(A)+O\left(\left(L^{k} \varepsilon\right)^{3+x_{0}}\right)\left|T_{1}^{(k)}\right|\right] .
\end{aligned}
$$

The integral above is calculated by the cumulant expansion up to the sixth order in $g_{k}$, the error being of the order $O\left(\left(L^{k} \varepsilon\right)^{3+\kappa_{0}}\right)\left|T_{1}^{(k)}\right|$. Lower order terms are represented by graphs with vertices determined by the terms in (56), and with lines corresponding to the propagator $C^{(k)}$.

We analyse the perturbative expressions in the same way as in the first step. We expand all the propagators into the generalized random walk expansions. We get a sum of terms, each having a localization domain $X$. Terms with localization domains $X$ having non-empty intersections with $\Omega_{k+1}^{c}$ are estimated by $O\left(g_{k}\right)\left|Z_{k}\right|$. Terms with domains $X$, which are not contained in a cube of the size $R\left(g_{k}\right) M_{1}$, are estimated by $O\left(\left(L^{k} \varepsilon\right)^{3+\kappa_{0}}\right)\left|T_{1}^{(k)}\right|$. The remaining terms give the sum

$$
\sum_{X} \mathscr{P}_{k+1}^{\prime}\left(g_{k}, X, U_{k+1}\right)
$$

over localizations $X \subset \Omega_{k+1}$, which are connected unions of big blocks, and which are contained in cubes of the size $R\left(g_{k}\right) M_{1}$. The terms $\mathscr{P}_{k+1}^{\prime}$ satisfy the bound (25) (with the indices 1,0 replaced by $k+1, k$ ), and are gauge invariant functions of $U_{k+1}$, in fact of an arbitrary gauge field configuration having regularity properties similar to the properties of $U_{k+1}$. We use results of Sect. $F[7]$, and we construct the 
representation $U_{k+1}=\exp i \eta \mathscr{H}(B)$ (modulo a gauge transformation) in a neighbourhood of $\square_{1}$. The configuration $B$ restricted to $\square_{1}^{(k+1)}=\square_{1} \cap T^{(k+1)}$ is given by the formula (27). We expand the function $\mathscr{P}_{k+1}^{\prime}\left(g_{k}, X\right.$, exp in $\left.\mathscr{H}(B)\right)$ with respect to $\mathscr{H}(B)$ at first, up to the sixth order, so we have the formula (30) (for $\eta$-scale). The same conclusion (32) holds for the first order functional derivative of $\mathscr{P}_{k+1}^{\prime}\left(g_{k}, X\right.$, exp in $\left.\mathscr{H}\right)$ at $\mathscr{H}=0$. Next we expand $\mathscr{H}(B)$ with respect to $B$, again up to the sixth order. Finally we estimate all terms with $B$ localized in $\square_{1}^{c}$ using the exponential decay of propagators. Summing up terms with the same monomial in $B$ yields the following analog of (33),

$$
\begin{aligned}
& \sum_{X} \mathscr{P}_{k+1}^{\prime}\left(g_{k}, X, U_{k+1}\right)=\sum_{X} \mathscr{P}_{k+1}^{\prime}\left(g_{k}, X, 1\right) \\
& \quad+\sum_{Y_{k+1}} \mathscr{P}_{k+1}\left(g_{k}, Y_{k+1}, U_{k+1}\right)+O\left(\left(L^{k} \varepsilon\right)^{3+\kappa_{0}}\right)\left|T_{1}^{(k)}\right|
\end{aligned}
$$

where the expressions above are as in (43), only $j$ and $k$ there are replaced by $k+1$.

Complementing the constants in (55) to the full lattice $T^{(k)}$, and gathering together all the transformations and estimates, we obtain the inductive inequality (41) for $k$ replaced by $k+1$, but with the additional term

$$
\log Z^{(k)}\left(B\left(\Lambda_{k+1}\right), U_{k+1}\right)-\log Z^{(k)}\left(B\left(\Lambda_{k+1}\right), 1\right) .
$$

The constant $E_{k+1}$ is defined as $E_{k+1}=E_{k}-E^{(k)}$, where

$$
E^{(k)}=\log \sigma_{0}\left|T_{1}^{(k) *}\right|+d(\mathrm{~g}) \log g_{k}\left|T_{1}^{(k) *}\right|+\log Z^{(k)}\left(T_{1}^{(k)}, 1\right)+\sum_{X} \mathscr{P}_{k+1}^{\prime}\left(g_{k}, X, 1\right)
$$

To complete the proof of the inductive assumption (41) we have to expand the term (61) analogously to (60). It can be localized in a similar way, although a bit more complicated, as the perturbative expressions. We write it as the logarithm of the Gaussian integral determined by the quadratic form $\left\langle A, \Delta_{k} A\right\rangle$ and the $\delta$-functions $\delta(Q A) \delta_{A x}(A)$. We eliminate the $\delta$-functions and we write the integral in terms of the independent variables $\tilde{A}$ introduced at the beginning of Sect. $E$ [5]. They are connected with $A$ by the simple linear operator $C$ described there, $A=C \tilde{A}$, and we obtain the Gaussian integral determined by the positive quadratic form $\left\langle A, C^{*} \Delta_{k} C A\right\rangle$. The elimination yields also a sum of local terms determined by the coefficient at the variable $A\left(b_{0}(c)\right)$ in $(Q A)(c)$. More exactly the coefficient is a linear operator $S\left(V_{k}^{(k)}, b_{0}(c)\right)$ acting on the Lie algebra $g$, see the formula (124) [4] for a precise definition, and we obtain the sum of terms $-\log \operatorname{det} S\left(V_{k}^{(k)}, b_{0}(c)\right)$. They are simple, local, gauge invariant functions of $V_{k}^{(k)}=\bar{U}_{k+1}^{k}$, and are analyzed in the same way as the perturbative expressions.

We consider the Gaussian integral now. Its logarithm is equal to a sum of an absolute constant, cancelled by the same constant from the second term in (61), and the expression

$$
\begin{aligned}
\frac{1}{2} \log \operatorname{det}\left(C^{*} \Delta_{k} C\right)^{-1} & =-\frac{1}{2} \operatorname{Tr} \log \left(C^{*} \Delta_{k} C\right) \\
& =-\frac{1}{4 \pi i} \int_{C} d z \log z \operatorname{Tr}\left(z I-C^{*} \Delta_{k} C\right)^{-1},
\end{aligned}
$$

where the contour $C$ is a union of the circle $|z|=\gamma_{1}$, and the two intervals $\left[2 \gamma_{1}, 0\right]$ 
with opposite orientations. The number $\gamma_{1}$ is an upper bound of the positive, bounded operator $C^{*} \Delta_{k} C$. Hence the integral above can be written as a sum of two integrals, and we have

$$
\begin{gathered}
\frac{1}{2} \log \operatorname{det}\left(C^{*} \Delta_{k} C\right)^{-1}=\frac{1}{2} \int_{0}^{2 \gamma_{1}} d x \operatorname{Tr}\left(C^{*} \Delta_{k} C+x I\right)^{-1}-\sum_{n=0}^{\infty} \frac{1}{4 \pi i} \int_{|z|=2 \gamma_{1}} d z \log z \frac{1}{z^{n+1}}\left(C^{*} \Delta_{k} C\right)^{n} \\
=\frac{1}{2} \int_{0}^{2 \gamma_{1}} \mathrm{dx} \operatorname{Tr}\left(C^{*} \Delta_{k} C+x I\right)^{-1}-\frac{1}{2} C^{*} \Delta_{k} C+\sum_{n=1}^{\infty} \frac{(-1)^{n}}{2 n}\left(2 \gamma_{1}\right)^{-n}\left(C^{*} \Delta_{k} C\right)^{n}
\end{gathered}
$$

The last sum above can be analyzed now in many ways, for example we may use the formula (3.156) [5] for the operator $\Delta_{k}$ and expand it into generalized random walks. This gives an expansion for the sum above into gauge invariant, localized expressions, with proper exponential decay properties. These are analyzed further in the same way as the perturbative terms. The operator under the integral has a representation similar to $\left(C^{*} \Delta_{k} C\right)^{-1}$. More exactly the operator $C\left(C^{*} \Delta_{k} C\right.$ $+x I)^{-1} C^{*}$ is represented by the integral (3.183) [5] with the additional term $-1 / 2 x\|\tilde{\chi}(Q A+\bar{D} \mu(Q A))\|^{2}$ under the exponential function, where $\tilde{\chi}$ is the characteristic function of the set of bonds corresponding to variables $\tilde{A}$. This term determines a non-negative, bounded and almost local operator. The integral yields the representation analogous to (3.185)

$$
\left(C^{*} \Delta_{k} C+x I\right)^{-1}=(I+\bar{D} \mu) Q \widetilde{G}_{3}(x) Q^{*}\left(I+\mu^{*} \bar{D}^{*}\right) \int_{B\left(\Lambda_{k+1}\right)},
$$

where $\widetilde{G}_{3}(x)$ is defined as $\widetilde{G}_{2}$, but with this additional operator. The operator $\widetilde{G}_{3}(x)$ has the same properties as $\widetilde{G}_{2}$, especially it can be represented by a generalized random walk expansion. By the above formula this gives an expansion of the last integral in (63) into a sum of gauge invariant, localized terms. They are again analyzed in the way described before. Now let us notice that gathering together the first terms in the expansions (30) we obtain the expansion of (63) for the external field $U_{k+1}=1$. This is cancelled by the second term in (61), and we obtain the desired expansion.

Thus we have estimated $\rho_{k+1}$ by an expression which is almost equal to the righthand side of the inductive assumption (41) for $k+1$. To get the exact inequality we estimate a sum of all terms $\mathscr{P}_{j}\left(Y_{j}, U_{k+1}\right)$ with localizations $Y_{j}$ not contained in $\Omega_{k+1}$ by $O(1)\left|\Lambda_{k}\right|$, or by $O(1)\left|Z_{k}\right|$.

The lower bound is proved in the same way, with all simplifications coming from the fact that $\Omega_{k+1}=T_{\eta}$.

Thus we have proved.

Theorem 2. The sequence of densities $\rho_{k}$ defined by the inductive equations (2), with $\rho_{0}$ given by (1), satisfies the inequalities (41), (47).

\section{Concluding Remarks}

We have to show that the inequalities (41), (47) imply Theorem 1, i.e. the inequalities (5). Let us consider the upper bound (41). We estimate the interaction terms using the bounds (44)-(46) by $O(1) M_{1}^{3} g_{k-1}^{2} p^{2}\left(g_{k-1}\right)\left|\Lambda_{k}\right| \leqq O(1)\left|T_{1}^{(k)}\right|$. The constant $E_{k}$ is given 
by the sum

$$
E_{k}=\sum_{j=k}^{K-1} E^{(j)},
$$

where $E^{(j)}$ is defined by (62). From (25), which holds for arbitrary $j$, we get easily $\left|E^{(j)}\right| \leqq O(1)\left|T_{1}^{(j)}\right|$, hence

$$
\left|E_{k}\right| \leqq \sum_{k=k}^{K-1} O(1)\left|T_{1}^{(j)}\right|=\sum_{j=k}^{K-1} O(1) L^{-3(j-k)}\left|T_{1}^{(k)}\right| \leqq O(1)\left|T_{1}^{(k)}\right| .
$$

We obtain a bound of the form (41) with the expression

$$
-\frac{1}{g_{k}^{2}} A^{\eta}\left(U_{k}\right)+\sum_{j=0}^{k-1} O\left(\log g_{j}^{-1}\right)\left|Z_{j}\right|+O(1)\left|T_{1}^{(k)}\right|,
$$

in the exponential. To prove the inequality (5) we have to produce all small factors connected with large fields regions $P$ in the functions $\zeta_{A_{j}}$. Let us take a plaquette $p^{\prime} \subset \Lambda_{j}$ and such that $\left|V_{j}\left(\partial p^{\prime}\right)-1\right| \geqq g_{j} p\left(g_{j}\right)$. We have

$$
\bar{U}_{k}^{j}=V_{j} \text { on } \Lambda_{j} \text {, }
$$

and the configuration $U_{k}$ satisfies the following regularity condition on $B^{j}\left(\Lambda_{j}\right)$.

$$
\left|U_{k}(\partial p)-1\right|<O(1) g_{j} p\left(q_{j}\right) L^{-2 j} .
$$

Applying the inequalities (50), (53) [4], we have

$$
\left|\bar{U}_{k}^{j}\left(\partial p^{\prime}\right)-1\right|<\sum_{x \in B^{\prime}\left(x_{0}\right)} L^{-3 i} \sum_{p \subset\left(p^{\prime}\right)_{x}}\left|U_{k}(\partial p)-1\right|+O(1)\left(g_{j} p\left(g_{j}\right)\right)^{2},
$$

where $p^{\prime}=\left\langle x_{0}, y_{0}, z_{0}, w_{0}\right\rangle,\left(p^{\prime}\right)_{x}$ denotes the plaquette $p^{\prime}$ transported parallelly to the point $x$, i.e. the lower left corner coincides with the point $x$. Squaring both sides of the above inequality and using (67) yields

$$
\begin{aligned}
\left|V_{j}\left(\partial p^{\prime}\right)-1\right|^{2} & <\sum_{x \in B^{3}\left(x_{0}\right)} L^{-j} \sum_{p \subset(p)_{x}}\left|U_{k}(\partial p)-1\right|^{2}+O(1)\left(g_{j} p\left(g_{j}\right)\right)^{3} \\
& \leqq 2 \sum_{p \in A^{\prime}} L^{j}\left[1-\operatorname{Retr} U_{k}(\partial p)\right]+O(1)\left(g_{j} p\left(g_{j}\right)\right)^{3},
\end{aligned}
$$

where $\Delta^{\prime}=B^{j}\left(x_{0}\right) \cup B^{j}\left(y_{0}\right) \cup B^{j}\left(z_{0}\right) \cup B^{j}\left(w_{0}\right)$. This inequality can be written finally as

$$
\begin{aligned}
\frac{1}{g_{k}^{2}} \sum_{p \in \Delta} \eta^{-1}\left[1-\operatorname{Retr} U_{k}(\partial p)\right] & \geqq \frac{1}{2 g_{j}^{2}}\left|V_{j}\left(\partial p^{\prime}\right)-1\right|^{2}-O(1) g_{j} p^{3}\left(g_{j}\right) \\
& \geqq \frac{1}{2} p^{2}\left(g_{j}\right)-O(1) g_{j} p^{3}\left(g_{j}\right) \geqq \frac{1}{4} p^{2}\left(g_{j}\right)
\end{aligned}
$$

for $g_{j}$ sufficiently small. Thus the part of the action $1 / g_{k}^{2} A^{\eta}\left(U_{k}\right)$ localized to the sum of four $j$-blocks $\Delta^{\prime}$ connected with the plaquette $p^{\prime}$ can be bounded from below by $1 / 4 p^{2}\left(g_{j}\right)$, and the corresponding part of the exponential gives the small factor $\exp \left(-1 / 4 p^{2}\left(g_{j}\right)\right)$. We get these small factors for all plaquettes in all large fields set $P$. Results related to stability bound (70) have been obtained by P. Federbush in $[17,18]$. The analysis of Sect. 3.C [9], which is model independent, show that these 
small factors are enough to control all sums in (41), together with the second term in (65). This gives the upper bound in (5). The lower bound is simpler, it is enough to use (44)-(46) and (66). Thus we have completed the proof of Theorem 1.

\section{References}

1. Balaban, T: Renormalization group methods in non-Abelian gauge theories. Harvard preprint HUTMP B134, or Recent results in constructing gauge fields. Physica 124A, 79-90 (1984)

2. Balaban, T.: Propagators and renormalization transformations for lattice gauge theories. I. Commun, Math. Phys. 95, 17-40 (1984)

3. Balaban, T.: Propagators and renormalization transformations for lattice gauge theories. II. Commun. Math. Phys, (in press)

4. Balaban, T.: Averaging operations for lattice gauge theories. Commun. Math. Phys. 98, 17-51 (1985)

5. Balaban, T.: Propagators for lattice gauge theories in a background field. Harvard preprint HUTMP B153. Commun. Math. Phys. 99, 389-434 (1985)

6. Balaban, T.: Spaces of regular gauge field configurations on a lattice and gauge fixing conditions. Commun. Math. Phys. 99, 75-102 (1985)

7. Balaban, $T$.: The variational problem and background fields in renormalization group method for lattice gauge theories. Commun. Math. Phys. (in press)

8. Balaban, T.: (Higgs) $)_{2,3}$ quantum fields in a finite volume. I. A lower bound. Commun. Math. Phys. 85, 603-636 (1982)

9. Balaban, T.: (Higgs) 2.3 quantum fields in a finite volume. II. An upper bound. Commun. Math. Phys. 86, 555-594 (1982)

10. Balaban, T.: (Higgs) $)_{2,3}$ quantum fields in a finite volume. III. Renormalization. Commun. Math. Phys. 88, 411-445 (1983)

11. Benfatto, G., Cassandro, M., Gallavotti, G., Nicolo, F., Olivieri, E., Presutti, E., Scaciatelli, E.: Some probabilistic techniques in field theory. Commun. Math. Phys. 59, 143-166 (1978)

12. Benfatto, G., Cassandro, M., Gallavotti, G., Nicolo, F., Olivieri, E., Presutti, E., Scaciatelli, E.: Ultraviolet stability in Euclidean scalar field theories. Commun. Math. Phys. 71, 95-130 (1980)

13. Brydges, D. Fröhlich, J., Seiler, E.: On the construction of quantized gauge fields. I. General results. Ann. Phys. 121, 227-284 (1979)

14. Brydges, D., Fröhlich, J., Seiler, E.: Construction of quantized gauge fields. II. Convergence of the lattice approximation. Commun. Math. Phys. 71, 159-205 (1980)

15. Brydges, D., Fröhlich, J., Seiler, E.: On the construction of quantized gauge fields. III. The twodimensional Abelian Higgs model without cutoffs. Commun. Math. Phys. 79, 353-399 (1981)

16. Challifour, J. L.: Self-adjointness of lattice Yang-Mills Hamiltonians and Kato's inequality with indefinite metric. Preprint of Indiana University

17. Federbush, P.: A phase cell approach to Yang-Mills theory. I. Small field modes. Preprint of University of Michigan

18. Federbush, P.: A phase cell approach to Yang-Mills theory. II. Stability, modified renormalization group transformations. Preprint of University of Michigan

19. Gawedzki, K., Kupiainen, A.: Rigorous renormalization group and asymptotic freedom. In Scaling and self-similarity in physics. Fröhlich, J. (ed.) pp. 227-262 Boston: Birkhäuser 1983

20. Glimm, J., Jaffe, A.: Positivity of the $\phi_{3}^{4}$ Hamiltonian. Fortschr. Phys. 21, 327-376 (1973)

21. Glimm, J., Jaffe, A.: Quantum physics: A functional point of view. Berlin, Heidelberg, New York: Springer 1981

22. Kadanoff, L. P.: The application of renormalization group techniques to quarks and strings. Rev. Mod. Phys. 49, 267-296 (1977)

23. Osterwalder, K., Seiler, E.: Gauge field theories on a lattice. Ann. Phys. 110, 440-471 (1978)

24. Seiler, E.: Gauge theories as a problem of constructive quantum field theory and statistical mechanics. Lecture Notes in Physics, Vol. 159, Berlin, Heidelberg, New York: Springer 1982 
25. Varadarajan, V. S.: Lie groups, Lie algebras and their representations. New York: Prentice-Hall 1979 26. Wilson, K. G.: Confinement of quarks. Phys. Rev. D10, 2445-2459 (1974)

27. Wilson, K. G.: Quantum chromodynamics on a lattice. In: Quantum field theory and statistical mechanics, Cargèse 1976. Lévy, M., Mitter, P. (eds.) pp. 143-172. New York: Plenum Press 1977

28. Wilson, K. G.: Monte Carlo calculations for the lattice gauge theory. In: Recent developments in gauge theories, 't Hooft, G., Itzykson, C., Jaffe, A., Lehmann, M., Mitter, P. K., Singer, I. M., Stora, R., (eds.) pp. 363-402. New York: Plenum Press 1980

Communicated by A. Jaffe

Received March 11, 1985; in revised form June 3, 1985 